Bull. Korean Math. Soc. 49 (2012), No. 2, pp. 319-327

http://dx.doi.org/10.4134/BKMS.2012.49.2.319

\title{
MATHEMATICAL ANALYSIS OF A MULTIFLUID INTERPENETRATION MIX MODEL
}

\author{
HYEONSEONG JiN
}

\begin{abstract}
The equations of a multifluid interpenetration mix model are analyzed. The model is an intermediate mix model in the sense that it is defined by partial pressures but only a single global pressure and a single global temperature. It none-the-less avoids the stability difficulty. It is shown that the model is hyperbolic so that it is mathematically stable.
\end{abstract}

\section{Introduction}

For many purpose, the mean flow properties of each fluid phase or species of multifluid flow are sufficient information and the detailed flow description provided by a finely resolved simulation is more than required. It is for this reason that the derivation of averaged equations is a popular study $[18,5,16]$. Averaging is one of the most basic tools to deal with multiscale science, whether in turbulence modeling, materials science, etc. The equations of multiphase flow are derived by averaging the microphysical equations. This process introduces an essential difficulty, the closure problem, to replace the unknown averages of nonlinear expressions by some expressions written in terms of the averaged variables, to yield a closed set of equations. Different choices of closures, appropriate for distinct flow regimes, contribute to the number of different multiphase flow equations.

Multiphase equations also differ in the variables selected for averaging. For the first order closures, as are generally considered for multiphase flow, these would be averages of the conserved variables (density, momentum, energy) of each species. Possible additional variables would be the volume fractions and surface areas. Reduced models result from enforced constraints among these variables, such as common temperatures, pressures, or velocities among the species. The fully reduced model, in which all temperatures, pressures

Received November 1, 2010.

2010 Mathematics Subject Classification. 76Txx, 76F25, 76Nxx, 76 Axx.

Key words and phrases. multiphase flow, averaged equations, hyperbolic models, stability, closures.

This work was supported by Basic Science Research Programs through the National Research Foundation of Korea(NRF) funded by the Ministry of Education, Science and Technology(MEST) (No. 2009-0069783 and No. 2010-0010164). 
and velocities are equilibrated, is known as atomic mix. We refer to models assuming equilibration among one or two of these variables as intermediate models, and the complete model, with a complete set of independent variables for each species is denoted the chunk mix model, or the complete first order multiphase closure.

In general, the intermediate models with pressure and possibly thermal equilibrium have two fundamental problems. They are mathematically and numerically unstable, with complex characteristics for time propagation. Thus, the models are physically unacceptable and lead to ill-posed problems. As normally considered, with regularizing terms, e.g. from viscosity for numerical stabilization, the magnitude of the coefficients governing the regularization is determined by numerical stability considerations rather than by physical measurements. The result is a possibly overly smoothed solution of a possibly overly diffusive model. An additional problem for these intermediate models is a loss of thermodynamic completeness for the individual species. This deficiency is accommodated by some assumption, for example of an isentropic equilibration to the thermodynamically constrained values. The thermodynamic assumptions of the intermediate models are rarely documented, and since they are intended for use in cases where entropy is not conserved, the resulting errors are also not generally examined. See the discussion in $[4,9]$. These problems not withstanding, intermediate models are widely used [19, 20, 7, 14].

In contrast to the single-pressure models $[17,13,11]$, the chunk mix twopressure models $[13,1,2,5]$ which have distinct phase pressures eliminate mathematical difficulties associated with single pressure flow models, leading to hyperbolic models. An independent evolution equation for the volume fraction is used as an alternative to setting an equilibrated pressure. The chunk mix models have been analyzed in $[3,6,8]$. They could be considered the full or complete first order multiphase closure, in that they contain in some sense the intermediate closures as limiting cases. It has been used in this sense to derive a two temperature closure which is velocity and pressure equilibrated [4]. The pressures of the chunk model are close to equilibration, but by not imposing this equality as an identity, it avoids some complications of mixed phase thermodynamics and issues of hyperbolic instability in the averaged equations, which would lead to a non-physical phase separation. The single pressure projection reduces the model to a system of single pressure equations. The constraint of pressure equilibrium between phases produces a boundary between hyperbolic and nonhyperbolic regions [10].

The interpenetration mix model proposed by Scannapieco and Cheng [15] is intermediate in the sense that it is defined by partial pressure but only a single global pressure and a single global temperature. It none-the-less avoids both difficulties of the intermediate models. We analyze the Scannapieco-Cheng model in a characteristic analysis. Main result is that this model is hyperbolic (mathematically and numerically stable) in the sense considered here, and each partial pressure is defined by the laws of thermodynamics for its own species. 
Thus the thermodynamics of this model is that of atomic mix. This analysis implies that the model containing two partial pressures results in a system which exhibits change of type (hyperbolic/nonhyperbolic) [11, 12].

\section{Analysis of the multifluid mix model}

Scannapieco and Cheng [15] have proposed a multifluid interpenetration mix model, in which the set of multifluid equations was derived rigorously from the collisional Boltzmann equation in a self-consistent manner. The model equations are mathematically complete and physically consistent with only one free parameter. Here we analyze the interpenetration mix model by a characteristic analysis.

For the multifluid interpenetration mix, we have the species equations

$$
\begin{aligned}
& \frac{\partial}{\partial t} \rho^{s}+\frac{\partial}{\partial x_{j}}\left(\rho^{s} v_{j}^{*}\right)+\frac{\partial}{\partial x_{j}}\left(\rho^{s}\left\langle U_{j}^{s}\right\rangle\right)=S_{\text {coll }}^{s} \\
& \frac{\partial}{\partial t}\left(\rho^{s}\left\langle U_{j}^{s}\right\rangle\right)+\frac{\partial}{\partial x_{i}}\left(v_{i}^{*} \rho^{s}\left\langle U_{j}^{s}\right\rangle\right)+\frac{\partial}{\partial x_{i}} \Psi_{i j}^{s} \\
& \quad+\rho^{s}\left(\frac{\partial}{\partial t} v_{j}^{*}+v_{i}^{*} \frac{\partial}{\partial x_{i}} v_{j}^{*}+\left\langle U_{i}^{s}\right\rangle \frac{\partial}{\partial x_{i}} v_{j}^{*}-\left\langle a_{j}^{s}\right\rangle\right)=\left(A_{j}^{s}\right)_{\text {coll }}, \\
& \frac{\partial}{\partial t} e^{s}+\frac{\partial}{\partial x_{j}}\left(v_{j}^{*} e^{s}\right)+\frac{\partial}{\partial x_{j}} Q_{j}^{s}+\Psi_{i j}^{s} \frac{\partial}{\partial x_{i}} v_{j}^{*} \\
& \quad+\rho^{s}\left\langle U_{j}^{s}\right\rangle\left(\frac{\partial}{\partial t} v_{j}^{*}+v_{i}^{*} \frac{\partial}{\partial x_{i}} v_{j}^{*}\right)-\rho^{s}\left\langle a_{j}^{s} U_{j}^{s}\right\rangle=E_{\mathrm{coll}}^{s}
\end{aligned}
$$

and the bulk fluid equations

$$
\begin{aligned}
& \frac{\partial}{\partial t} \rho^{*}+\frac{\partial}{\partial x_{j}}\left(\rho^{*} v_{j}^{*}\right)=0, \\
& \rho^{*}\left(\frac{\partial}{\partial t} v_{j}^{*}+v_{i}^{*} \frac{\partial}{\partial x_{i}} v_{j}^{*}\right)+\frac{\partial}{\partial x_{i}} \Psi_{i j}^{*}-\rho^{*} a_{j}^{*}=0, \\
& \frac{\partial}{\partial t} e^{*}+\frac{\partial}{\partial x_{j}}\left(e^{*} v_{j}^{*}\right)+\frac{\partial}{\partial x_{j}} Q_{j}^{*}+\Psi_{i j}^{*} \frac{\partial}{\partial x_{i}} v_{j}^{*}=\sum_{s} \rho^{s}\left\langle a_{j}^{s} U_{j}^{s}\right\rangle,
\end{aligned}
$$

for the species mean-mass density $\rho^{s}$, bulk flow mass density $\rho^{*} \equiv \sum_{s} \rho^{s}$, mean-mass weighted bulk flow velocity $v_{j}^{*}$, peculiar velocity $\left\langle U_{j}^{s}\right\rangle$, species total kinetic energy density $e^{s} \equiv \frac{1}{2} \rho^{s}\left\langle U_{j}^{s} U_{j}^{s}\right\rangle$ and total mass-mean energy density $e^{*} \equiv \sum_{s} e^{s}$, where $a_{j}$ is an external acceleration. A constant $a_{j}$ corresponds to the Rayleigh-Taylor mixing and a shock or an impulsive $a_{j}=\delta(t)$ describes Richtmyer-Meshkov mixing. Here

$$
\Psi_{i j}^{*} \equiv \sum_{s} \Psi_{i j}^{s}, \quad \Psi_{i j}^{s} \equiv \rho^{s}\left\langle U_{i}^{s} U_{j}^{s}\right\rangle
$$


represent, respectively, the total stress tensor of the bulk fluid and the total stress tensor of species $s$ in a frame moving with the mean mass velocity and

$$
Q_{j}^{*} \equiv \sum_{s} Q_{j}^{s}, \quad Q_{j}^{s} \equiv \frac{1}{2} \rho^{s}\left\langle U_{j}^{s} U_{i}^{s} U_{i}^{s}\right\rangle
$$

are, respectively, the total mass mean energy flux density of the bulk fluid and the total energy flux density for species $s$. The collision terms are

$$
\begin{aligned}
S_{\text {coll }}^{s} & =\int M^{s}\left(\frac{\delta f^{s}}{\delta t}\right)_{\text {coll }} d^{3} v^{s}, \\
\left(A_{j}^{s}\right)_{\text {coll }} & =\int M^{s} U_{j}^{s}\left(\frac{\delta f^{s}}{\delta t}\right)_{\text {coll }} d^{3} v^{s}, \\
E_{\text {coll }}^{s} & =\int \frac{1}{2} M^{s} U_{j}^{s} U_{j}^{s}\left(\frac{\delta f^{s}}{\delta t}\right)_{\text {coll }} d^{2} v^{s} .
\end{aligned}
$$

We denote the average of a physical quantity $\phi^{s}$ of species $s$ by

$$
\left\langle\phi^{s}\right\rangle \equiv \frac{1}{N^{s}} \int \phi^{s} f^{s} d^{3} v^{s} .
$$

Here $N^{s}$ is the total number density of particles of species $s$ defined as

$$
N^{s} \equiv \int f^{s} d^{3} v^{s}
$$

where $f\left(t, x_{j}, v_{j}\right)$ is the single-particle distribution function which represents the number density of particles found in an infinitesimal volume of phase space centered on the phase space point $\left(x_{j}, v_{j}\right)$ at time $t$ and satisfies the collisional Boltzmann equation

$$
\frac{\partial f}{\partial t}+v_{j} \frac{\partial f}{\partial x_{j}}+a_{j} \frac{\partial f}{\partial v_{j}}=\left(\frac{\delta f}{\delta t}\right)_{\text {coll }} .
$$

The species velocity $v_{j}^{s}$ is written as a sum of its peculiar (fluctuating) velocity $U_{j}^{s}$ and the mean-mass bulk flow velocity $v_{j}^{*}$,

$$
v_{j}^{s} \equiv U_{j}^{s}+v_{j}^{*}, \quad v_{j}^{*} \equiv \frac{\sum_{s} N^{s} M^{s}\left\langle v_{j}^{s}\right\rangle}{\sum_{s} N^{s} M^{s}},
$$

where $M^{s}$ is the mass of a particle of species $s$. Using (15), the species and bulk fluid equations (1)-(6) have been derived by transformation of variable set from $\left(t, x_{j}, v_{j}^{s}\right)$ to $\left(t, x_{j}, v_{j}^{*}, U_{j}^{s}\right)$. The system of the equations results in a $9 \times 9$ system for the species $s=1,2$. The split of the species velocity makes the characteristic analysis of the system complicated. Thus we derive the simplified equations by undoing the split of the species velocity.

We average the collisional Boltzmann equation (14) multiplied by any physical quantity $\phi^{s}$ of species $s$ by integration over velocity space. Then we obtain 
the averaged equation of any physical quantity $\phi^{s}$ as follows:

$$
\begin{aligned}
& \frac{\partial}{\partial t}\left(N^{s}\left\langle\phi^{s}\right\rangle\right)+\frac{\partial}{\partial x_{i}}\left(N^{s}\left\langle v_{i}^{s} \phi^{s}\right\rangle\right) \\
& -N^{s}\left[\left\langle\frac{\partial \phi^{s}}{\partial t}\right\rangle+\left\langle v_{i}^{s} \frac{\partial \phi^{s}}{\partial x_{i}}\right\rangle+\left\langle a_{i}^{s} \frac{\partial \phi^{s}}{\partial v_{i}}\right\rangle\right]=\int \phi^{s}\left(\frac{\delta f^{s}}{\delta t}\right)_{\text {coll }} d^{3} v .
\end{aligned}
$$

We note that $\partial v_{j}^{s} / \partial t, \partial v_{j}^{s} / \partial x_{i}$ are zero since $x_{j}, v_{j}^{s}, t$ are independent variables. From (15), notice that

$$
v_{j}^{s}-\left\langle v_{j}^{s}\right\rangle=U_{j}^{s}-\left\langle U_{j}^{s}\right\rangle .
$$

We now derive the momentum equation by applying the quantity $\phi^{s}=$ $M^{s} v_{j}^{s}$. Then Eq.(16) becomes

$$
\frac{\partial}{\partial t}\left(\rho^{s}\left\langle v_{j}^{s}\right\rangle\right)+\frac{\partial}{\partial x_{i}}\left(\rho^{s}\left\langle v_{i}^{s} v_{j}^{s}\right\rangle\right)=\rho^{s}\left\langle a_{j}^{s}\right\rangle+\left(A_{j}^{s}\right)_{\text {coll }}+v_{j}^{*} S_{\text {coll }}^{s} .
$$

From (17) and the closure relationships, one calculation shows

$$
\begin{aligned}
\rho^{s}\left\langle v_{i}^{s} v_{j}^{s}\right\rangle & =\rho^{s}\left\langle\left(v_{i}^{s}-\left\langle v_{i}^{s}\right\rangle+\left\langle v_{i}^{s}\right\rangle\right)\left(v_{j}^{s}-\left\langle v_{j}^{s}\right\rangle+\left\langle v_{j}^{s}\right\rangle\right)\right\rangle \\
& =\rho^{s}\left\langle v_{i}^{s}\right\rangle\left\langle v_{j}^{s}\right\rangle+\rho^{s}\left\langle\left(v_{i}^{s}-\left\langle v_{i}^{s}\right\rangle\right)\left(v_{j}^{s}-\left\langle v_{j}^{s}\right\rangle\right)\right\rangle \\
& =\rho^{s}\left\langle v_{i}^{s}\right\rangle\left\langle v_{j}^{s}\right\rangle+\rho^{s}\left\langle\left(U_{i}^{s}-\left\langle U_{i}^{s}\right\rangle\right)\left(U_{j}^{s}-\left\langle U_{j}^{s}\right\rangle\right)\right\rangle \\
& =\rho^{s}\left\langle v_{i}^{s}\right\rangle\left\langle v_{j}^{s}\right\rangle+P_{i j}^{s},
\end{aligned}
$$

where the thermal stress tensor $P_{i j}^{s}$ of species $s$ is written as in the form

$$
\begin{aligned}
P_{i j}^{s} & \equiv \rho^{s}\left\langle\left(U_{i}^{s}-\left\langle U_{i}^{s}\right\rangle\right)\left(U_{j}^{s}-\left\langle U_{j}^{s}\right\rangle\right)\right\rangle=P^{s} \delta_{i j}+\Pi_{i j}^{s} \\
& =\rho^{s}\left\langle\left(v_{i}^{s}-\left\langle v_{i}^{s}\right\rangle\right)\left(v_{j}^{s}-\left\langle v_{j}^{s}\right\rangle\right)\right\rangle,
\end{aligned}
$$

$P^{s}$ represents the scalar pressure of species $s, \delta_{i j}$ is the Kronecker delta, and $\Pi_{i j}^{s}$ is the viscous stress tensor of species $s$. We observe that

$$
P_{i j}^{*} \equiv \sum_{s} P_{i j}^{s} \equiv P^{*} \delta_{i j}+\Pi_{i j}^{*}, P^{*}=\sum_{s} P^{s}, \Pi_{i j}^{*}=\sum_{s} \Pi_{i j}^{s}
$$

represent the corresponding quantities for the bulk fluid mixture. Specifically, the mean global pressure $P^{*}$ exists in the bulk fluid equations (5) and (6). Using Eqs.(18) and (19), we obtain the momentum equation

(22) $\frac{\partial}{\partial t}\left(\rho^{s}\left\langle v_{j}^{s}\right\rangle\right)+\frac{\partial}{\partial x_{i}}\left(\rho^{s}\left\langle v_{i}^{s}\right\rangle\left\langle v_{j}^{s}\right\rangle\right)+\frac{\partial}{\partial x_{i}} P_{i j}^{s}=\rho^{s}\left\langle a_{j}^{s}\right\rangle+\left(A_{j}^{s}\right)_{\text {coll }}+v_{j}^{*} S_{\text {coll }}^{s}$. 
Applying $\phi^{s}=\frac{1}{2} M^{s} v_{j}^{s} v_{j}^{s}$ to Eq.(16), we derive the energy equation. Then

$$
\begin{aligned}
& \frac{\partial}{\partial t}\left(\frac{1}{2} \rho^{s}\left\langle v_{j}^{s} v_{j}^{s}\right\rangle\right)+\frac{\partial}{\partial x_{i}}\left(\frac{1}{2} \rho^{s}\left\langle v_{i}^{s} v_{j}^{s} v_{j}^{s}\right\rangle\right)-\rho^{s}\left\langle a_{j} v_{j}^{s}\right\rangle \\
= & \frac{1}{2} \int M^{s} v_{j}^{s} v_{j}^{s}\left(\frac{\delta f^{s}}{\delta t}\right)_{\text {coll }} d^{3} v .
\end{aligned}
$$

One calculates the identities

$$
\begin{aligned}
\frac{1}{2} \rho^{s}\left\langle v_{j}^{s} v_{j}^{s}\right\rangle & =\frac{1}{2} \rho^{s}\left\langle\left(v_{j}^{s}-\left\langle v_{j}^{s}\right\rangle+\left\langle v_{j}^{s}\right\rangle\right)\left(v_{j}^{s}-\left\langle v_{j}^{s}\right\rangle+\left\langle v_{j}^{s}\right\rangle\right)\right\rangle \\
& =\frac{1}{2} \rho^{s}\left\langle v_{j}^{s}\right\rangle\left\langle v_{j}^{s}\right\rangle+\frac{1}{2} \rho^{s}\left\langle\left(v_{j}^{s}-\left\langle v_{j}^{s}\right\rangle\right)\left(v_{j}^{s}-\left\langle v_{j}^{s}\right\rangle\right)\right\rangle \\
& =\frac{1}{2} \rho^{s}\left\langle v_{j}^{s}\right\rangle\left\langle v_{j}^{s}\right\rangle+\frac{1}{2} \rho^{s}\left\langle\left(U_{j}^{s}-\left\langle U_{j}^{s}\right\rangle\right)\left(U_{j}^{s}-\left\langle U_{j}^{s}\right\rangle\right)\right\rangle \\
& =\frac{1}{2} \rho^{s}\left\langle v_{j}^{s}\right\rangle\left\langle v_{j}^{s}\right\rangle+e_{T}^{s},
\end{aligned}
$$

$$
\begin{aligned}
\frac{1}{2} \rho^{s}\left\langle v_{i}^{s} v_{j}^{s} v_{j}^{s}\right\rangle= & \frac{1}{2} \rho^{s}\left\langle\left(v_{i}^{s}-\left\langle v_{i}^{s}\right\rangle+\left\langle v_{i}^{s}\right\rangle\right) v_{j}^{s} v_{j}^{s}\right\rangle \\
= & \frac{1}{2} \rho^{s}\left\langle v_{i}^{s}\right\rangle\left\langle v_{j}^{s} v_{j}^{s}\right\rangle+\frac{1}{2} \rho^{s}\left\langle\left(v_{i}^{s}-\left\langle v_{i}^{s}\right\rangle\right) v_{j}^{s} v_{j}^{s}\right\rangle \\
= & \left\langle v_{i}^{s}\right\rangle\left(\frac{1}{2} \rho^{s}\left\langle v_{j}^{s}\right\rangle\left\langle v_{j}^{s}\right\rangle+e_{T}^{s}\right) \\
& +\frac{1}{2} \rho^{s}\left\langle\left(v_{i}^{s}-\left\langle v_{i}^{s}\right\rangle\right)\left(v_{j}^{s}-\left\langle v_{j}^{s}\right\rangle+\left\langle v_{j}^{s}\right\rangle\right)\left(v_{j}^{s}-\left\langle v_{j}^{s}\right\rangle+\left\langle v_{j}^{s}\right\rangle\right)\right\rangle \\
= & \left\langle v_{i}^{s}\right\rangle\left(\frac{1}{2} \rho^{s}\left\langle v_{j}^{s}\right\rangle\left\langle v_{j}^{s}\right\rangle+e_{T}^{s}\right)+\rho^{s}\left\langle v_{j}^{s}\right\rangle\left\langle\left(v_{i}^{s}-\left\langle v_{i}^{s}\right\rangle\right)\left(v_{j}^{s}-\left\langle v_{j}^{s}\right\rangle\right)\right\rangle \\
& +\frac{1}{2} \rho^{s}\left\langle\left(v_{i}^{s}-\left\langle v_{i}^{s}\right\rangle\right)\left(v_{j}^{s}-\left\langle v_{j}^{s}\right\rangle\right)\left(v_{j}^{s}-\left\langle v_{j}^{s}\right\rangle\right)\right\rangle \\
= & \left\langle v_{i}^{s}\right\rangle\left(\frac{1}{2} \rho^{s}\left\langle v_{j}^{s}\right\rangle\left\langle v_{j}^{s}\right\rangle+e_{T}^{s}\right)+\left\langle v_{j}^{s}\right\rangle P_{i j}^{s} \\
& +\frac{1}{2} \rho^{s}\left\langle\left(U_{i}^{s}-\left\langle U_{i}^{s}\right\rangle\right)\left(U_{j}^{s}-\left\langle U_{j}^{s}\right\rangle\right)\left(U_{j}^{s}-\left\langle U_{j}^{s}\right\rangle\right)\right\rangle \\
= & \left\langle v_{i}^{s}\right\rangle\left(\frac{1}{2} \rho^{s}\left\langle v_{j}^{s}\right\rangle\left\langle v_{j}^{s}\right\rangle+e_{T}^{s}\right)+\left\langle v_{j}^{s}\right\rangle P_{i j}^{s}+Q_{T i}^{s} .
\end{aligned}
$$

Here the thermal kinetic energy, $e_{T}^{s}$, of species $s$ due to the species random and chaotic motion is defined by

$$
e_{T}^{s} \equiv \frac{1}{2} \rho^{s}\left\langle\left(U_{j}^{s}-\left\langle U_{j}^{s}\right\rangle\right)\left(U_{j}^{s}-\left\langle U_{j}^{s}\right\rangle\right)\right\rangle
$$




$$
=\frac{1}{2} \rho^{s}\left\langle\left(v_{j}^{s}-\left\langle v_{j}^{s}\right\rangle\right)\left(v_{j}^{s}-\left\langle v_{j}^{s}\right\rangle\right)\right\rangle
$$

and the heat flux

$$
\begin{aligned}
Q_{T i}^{s} & \equiv \frac{1}{2} \rho^{s}\left\langle\left(U_{i}^{s}-\left\langle U_{i}^{s}\right\rangle\right)\left(U_{j}^{s}-\left\langle U_{j}^{s}\right\rangle\right)\left(U_{j}^{s}-\left\langle U_{j}^{s}\right\rangle\right)\right\rangle \\
& =\frac{1}{2} \rho^{s}\left\langle\left(v_{i}^{s}-\left\langle v_{i}^{s}\right\rangle\right)\left(v_{j}^{s}-\left\langle v_{j}^{s}\right\rangle\right)\left(v_{j}^{s}-\left\langle v_{j}^{s}\right\rangle\right)\right\rangle
\end{aligned}
$$

due to the random and chaotic motions of species $s$ relative to its mean-mass velocity is written as

$$
Q_{T i}^{s}=-\kappa^{s} \frac{\partial}{\partial x_{i}} T^{s},
$$

where $\kappa^{s}$ is the coefficient of thermal conduction of species $s$. Substituting (24) and (25) into (23) and using the equation

$$
\begin{aligned}
& \frac{\partial}{\partial t}\left(\frac{1}{2} \rho^{s}\left\langle v_{j}^{s}\right\rangle\left\langle v_{j}^{s}\right\rangle\right)+\frac{\partial}{\partial x_{i}}\left(\frac{1}{2} \rho^{s}\left\langle v_{i}^{s}\right\rangle\left\langle v_{j}^{s}\right\rangle\left\langle v_{j}^{s}\right\rangle\right)+\left\langle v_{j}^{s}\right\rangle \frac{\partial}{\partial x_{i}} P_{i j}^{s} \\
= & \rho^{s}\left\langle a_{j}^{s}\right\rangle\left\langle v_{j}^{s}\right\rangle+\left\langle v_{j}^{s}\right\rangle\left[\left(A_{j}^{s}\right)_{\text {coll }}-\left\langle U_{j}^{s}\right\rangle S_{\text {coll }}^{s}+\frac{1}{2}\left\langle v_{j}^{s}\right\rangle S_{\text {coll }}^{s}\right]
\end{aligned}
$$

which is derived from (1) and (22), we obtain the energy equation

$$
\begin{aligned}
& \frac{\partial e_{T}^{s}}{\partial t}+\frac{\partial}{\partial x_{i}}\left(\left\langle v_{i}^{s}\right\rangle e_{T}^{s}\right)+P_{i j}^{s} \frac{\partial}{\partial x_{i}}\left\langle v_{j}^{s}\right\rangle+\frac{\partial}{\partial x_{i}} Q_{T i}^{s} \\
= & \rho^{s}\left(\left\langle a_{j}^{s} v_{j}^{s}\right\rangle-\left\langle a_{j}^{s}\right\rangle\left\langle v_{j}^{s}\right\rangle\right)+E_{\text {coll }}^{s}-\left\langle U_{j}^{s}\right\rangle\left(\left(A_{j}^{s}\right)_{\text {coll }}-\frac{1}{2}\left\langle U_{j}^{s}\right\rangle S_{\text {coll }}^{s}\right)
\end{aligned}
$$

for $e_{T}^{s}$.

We observe from (22) and (30) that the pressure has in it less than what was assumed in $[13,17]$. As a result, the momentum and energy equations couple only through non differential source terms, which have no role in the hyperbolicity. The hyperbolicity reduces to that for each fluid considered separately. A further consequence is that the source term in the model equations has more in it. Thus modeling of this term is of increased importance. The differences in the pressure in $[15]$ and $[13,17]$ are in fact subtle. We remark that Eqs.(22) and (30) can also be obtained by the modification of (2) and (3) by use of (15), (17), (1).

Assuming inviscid flow, i.e., $Q_{T i}^{s}=0$ and $P_{i j}^{s}=P^{s} \delta_{i j}$, suppressing the subscripts $i$ and $j$, from (1), (22) and (30), we obtain one dimensional multiphase flow equations which can be written as the form

$$
\frac{\partial W^{s}}{\partial t}+A \frac{\partial W^{s}}{\partial x}=G
$$


for $W^{s}$, where

$$
\begin{array}{r}
W^{s}=\left(\begin{array}{c}
\rho^{s} \\
\left\langle v^{s}\right\rangle \\
e_{T}^{s}
\end{array}\right), \quad A=A\left(W^{s}\right)=\left(\begin{array}{ccc}
\left\langle v^{s}\right\rangle & \rho^{s} & 0 \\
\frac{1}{\rho^{s}} \frac{\partial P^{s}}{\partial \rho^{s}} & \left\langle v^{s}\right\rangle & \frac{1}{\rho^{s}} \frac{\partial P^{s}}{\partial e_{T}^{s}} \\
0 & e_{T}^{s}+P^{s} & \left\langle v^{s}\right\rangle
\end{array}\right), \\
S_{\text {coll }}^{s} \\
G=\left(\begin{array}{c}
\left\langle a^{s}\right\rangle+\frac{1}{\rho^{s}}\left[A_{\text {coll }}^{s}-\left\langle U^{s}\right\rangle S_{\text {coll }}^{s}\right] \\
\left\langle a^{s} v^{s}\right\rangle-\left\langle a^{s}\right\rangle\left\langle v^{s}\right\rangle+\frac{1}{\rho^{s}}\left[E_{\text {coll }}^{s}-\left\langle U^{s}\right\rangle\left(A_{\text {coll }}^{s}-\frac{1}{2}\left\langle U^{s}\right\rangle S_{\text {coll }}^{s}\right)\right]
\end{array}\right) .
\end{array}
$$

The characteristic analysis gives the result as the following:

Theorem 2.1. The system of the multifluid interpenetration mix model equations (1), (22) and (30) is mathematically stable with real characteristics for time propagation.

Proof. The system (31) has eigenvalues

$$
\left\langle v^{s}\right\rangle, \quad\left\langle v^{s}\right\rangle \pm \sqrt{\frac{\partial P^{s}}{\partial \rho^{s}}+\frac{e_{T}^{s}+P^{s}}{\rho^{s}} \frac{\partial P^{s}}{\partial e_{T}^{s}}}
$$

and it is hyperbolic since $\frac{\partial P^{s}}{\partial \rho^{s}}+\frac{e_{T}^{s}+P^{s}}{\rho^{s}} \frac{\partial P^{s}}{\partial e_{T}^{s}}$ is positive.

\section{Conclusion}

In this paper, we have analyzed the equations of the multifluid interpenetration mix model. The model is an intermediate mix model in the sense that it is defined by partial pressures but only a single global pressure and a single global temperature. Nevertheless, this model shows hyperbolicity associated with chunk mix two-pressure models and avoids the mathematical difficulty associated with single pressure models. The thermodynamics of this model is that of atomic mix since each partial pressure is defined by the laws of thermodynamics for its own species. Thus, the interpenetration mix model contains favorable aspects of the intermediate, chunk, atomic mix models.

\section{References}

[1] M. Baer and J. Nunziato, A two-phase mixture theory for the deflagration-to-detonation transition $(D D T)$ in reactive granular materials, Int. J. Multiphase Flows 12 (1986), 861-889.

[2] Y. Chen, J. Glimm, D. H. Sharp, and Q. Zhang, A two-phase flow model of the RayleighTaylor mixing zone, Phys. Fluids 8 (1996), no. 3, 816-825.

[3] B. Cheng, J. Glimm, D. Saltz, and D. H. Sharp, Boundary conditions for a two pressure two phase flow model, Physica D 133 (1999), no. 1-4, 84-105.

[4] B. Cheng, J. Glimm, and D. H. Sharp, Multi-temperature multiphase flow model, Z. Angew. Math. Phys. 53 (2002), no. 2, 211-238. 
[5] D. A. Drew, Mathematical modeling of two-phase flow, Ann. Rev. Fluid Mech. 15 (1983), 261-291.

[6] J. Glimm, H. Jin, M. Laforest, F. Tangerman, and Y. Zhang, A two pressure numerical model of two fluid mixing, Multiscale Model. Simul. 1 (2003), no. 3, 458-484.

[7] F. Harlow and A. Amsden, Flow of interpenetrating material phases, J. Comput. Phys. 18 (1975), 440-464.

[8] H. Jin, J. Glimm, and D. H. Sharp, Compressible two-pressure two-phase flow models, Phys. Lett. A 353 (2006), 469-474.

[9] H. Jin, A study of multi-phase flow models, Indian J. Pure Appl. Math. 40 (2009), no. 3, 201-219.

[10] B. Keyfitz, Admissibility conditions for shocks in conservation laws that change type, SIAM J. Math. Anal. 22 (1991), no. 5, 1284-1292.

[11] Change of type in simple models of two-phase flow, In M. Shearer, editor, Viscous Profiles and Numerical Approximation of Shock Waves, pages 84-104. SIAM, Philadelphia, PA, 1991.

[12] _ Mathematical properties of nonhyperbolic models for incompressible two-phase flow, Proceedings of the 4th International Conference on Multiphase Flow, New Orleans, LA, 2001, submitted.

[13] V. H. Ransom and D. L. Hicks, Hyperbolic two-pressure models for two-phase flow, J. Comput. Phys. 53 (1984), no. 1, 124-151.

[14] R. Saurel and O. LeMetayer, A multiphase model for compressible flows with interfaces, shocks, detonation waves and cavitation, J. Fluid. Mech. 431 (2001), 239-271.

[15] A. Scannapieco and B. Cheng, A multifluid interpenetration mix model, Phys. Lett. A 299 (2002), 49-64.

[16] S. L. Soo, Particulates and Continuum: Multiphase Fluid Dynamics, Hemisphere, New York, 1989.

[17] H. B. Stewart and B. Wendroff, Two-phase flow: Models and methods, J. Comput. Phys. 56 (1984), no. 3, 363-409.

[18] G. Wallis, One-Dimensional Two-Phase Flow, McGraw-Hill, New York, 1969.

[19] D. L. Youngs, Numerical simulation of turbulent mixing by Rayleigh-Taylor instability, Physica D 12 (1984), 32-44.

[20] _ Modeling turbulent mixing by Rayleigh-Taylor instability, Physica D 37 (1989), 270-287.

Department of Mathematics

Jeju National University

JEJU 690-756, KorEA

E-mail address: hjin@jejunu.ac.kr 